\title{
COMPLEMENTARITY CONSTRAINT QUALIFICATION VIA THE THEORY OF 2-REGULARITY*
}

\author{
A. F. IZMAILOV ${ }^{\dagger}$ AND M. V. SOLODOV $\ddagger$
}

\begin{abstract}
We exhibit certain second-order regularity properties of parametric complementarity constraints, which are notorious for being irregular in the classical sense. Our approach leads to a constraint qualification in terms of 2-regularity of the mapping corresponding to the subset of constraints which must be satisfied as equalities around the given feasible point, while no qualification is required for the rest of the constraints. Under this 2-regularity assumption, we derive constructive sufficient conditions for tangent directions to feasible sets defined by complementarity constraints. A special form of primal-dual optimality conditions is also obtained. We further show that our 2-regularity condition always holds under the piecewise Mangasarian-Fromovitz constraint qualification, but not vice versa. Relations with other constraint qualifications and optimality conditions are also discussed. It is shown that our approach can be useful when alternative ones are not applicable.
\end{abstract}

Key words. equilibrium constraints, tangent cone, constraint qualification, 2-regularity, optimality conditions

AMS subject classifications. 90C30, 90C33

PII. S1052623499365292

1. Introduction. This paper is devoted to the analysis of local structure of a set defined by parametric complementarity constraints, such as

$$
D:=\left\{(x, y) \in \Re^{n} \times \Re^{m} \mid g(x, y) \geq 0, y \geq 0,\langle g(x, y), y\rangle=0\right\},
$$

where $g: \Re^{n} \times \Re^{m} \rightarrow \Re^{m}$, and $\langle\cdot, \cdot\rangle$ denotes the usual inner product in an appropriate space. The geometry of sets having this (or similar) structure is important in connection with mathematical programs with complementarity constraints, where $D$ appears as the feasible region:

$$
\begin{array}{ll}
\operatorname{minimize} & f(x, y) \\
\text { subject to } & (x, y) \in D .
\end{array}
$$

Mathematical programs with equilibrium constraints (MPEC), of which (1.2) is a special case, are a relatively new active field of research. We refer the reader to the monograph [10] for further references. It is known [4], and not difficult to see, that for $D$ given by (1.1) the classical Mangasarian-Fromovitz constraint qualification (MFCQ) [13] does not hold at any feasible point $(x, y) \in D$. Arguably, this is the single most important reason that makes treatment of this problem considerably more difficult when compared to regular (i.e., satisfying some standard constraint qualifications) nonlinear programs. Specifically, the nonsatisfaction of classical constraint qualifications makes a simple constructive description of the tangent cone to the set

* Received by the editors December 13, 1999; accepted for publication (in revised form) February 11, 2002; published electronically September 24, 2002.

http://www.siam.org/journals/siopt/13-2/36529.html

${ }^{\dagger}$ Computing Center of the Russian Academy of Sciences, Vavilova Street 40, Moscow, GSP-1, Russia (izmaf@ccas.ru). This author's research was supported by the Russian Foundation for Basic Research grants 99-01-00472 and 01-01-00810. The author also thanks IMPA, where he was a visiting professor during the completion of this work.

${ }^{\ddagger}$ Instituto de Matemática Pura e Aplicada, Estrada Dona Castorina 110, Jardim Botânico, Rio de Janeiro, RJ 22460-320, Brazil (solodov@impa.br). This author's research was supported in part by CNPq grant 300734/95-6, by PRONEX-Optimization, and by FAPERJ. 
$D$ (and its dual) difficult. This rules out standard stationarity/optimality conditions. The latter, in turn, makes it problematic to come up with reliable computational algorithms for solving MPEC.

To deal with these issues, a number of approaches have been proposed, among which are $[11,10,18,15,14,20]$. In this paper, we study complementarity constraints from the point of view of the theory of 2-regularity of twice differentiable mappings, which has not been used in this context previously. Our analysis clarifies the role that 2-regularity can play for MPEC, as well as provides an alternative view of some issues related to MPEC constraint qualifications and optimality conditions. We also exhibit some situations when other approaches do not apply but our results appear useful to characterize optimality.

Given any $\left(x^{*}, y^{*}\right) \in D$, define the three index sets

$$
\begin{aligned}
& I_{0}:=\left\{i \mid y_{i}^{*}=0=g_{i}\left(x^{*}, y^{*}\right)\right\}, \\
& I_{g}:=\left\{i \mid y_{i}^{*}>0=g_{i}\left(x^{*}, y^{*}\right)\right\}, \\
& I_{y}:=\left\{i \mid y_{i}^{*}=0<g_{i}\left(x^{*}, y^{*}\right)\right\},
\end{aligned}
$$

with $I_{0}$ being the degenerate set. Note that these index sets depend on the point under consideration. Since this point will be fixed throughout our analysis, we shall omit this dependency in our notation. Note also that locally, the constraints $g_{i}(x, y) \geq 0, i \in I_{y}$, are never active, and we can further deal with constraints $y_{i}=0, i \in I_{y}$, explicitly by eliminating the variables. Thus, to simplify the notation, we can assume that

$$
I_{y}=\emptyset \text {. }
$$

It is further easy to see that there exists a neighborhood $\mathcal{V}$ of $\left(x^{*}, y^{*}\right) \in D$ such that $D \cap \mathcal{V}=D^{*} \cap \mathcal{V}$, where

$$
D^{*}:=\left\{\begin{array}{l|l}
(x, y) & \begin{array}{l}
y_{i} \geq 0, g_{i}(x, y) \geq 0, y_{i} g_{i}(x, y)=0, i \in I_{0} \\
g_{i}(x, y)=0, i \in I_{g}
\end{array}
\end{array}\right\} .
$$

The sets $D$ and $D^{*}$ are therefore locally equivalent, and we can work with the representation given by (1.3). Let us define the mapping $F: \Re^{n} \times \Re^{m} \rightarrow \Re^{\left|I_{0}\right|+\left|I_{g}\right|}$ associated with equality constraints in $D^{*}$ :

$$
F_{i}(x, y):= \begin{cases}y_{i} g_{i}(x, y), & i \in I_{0} \\ g_{i}(x, y), & i \in I_{g}\end{cases}
$$

In what follows, we show that certain (second-order) regularity properties of this mapping $F$ are sufficient to completely characterize tangent directions to the set $D$ and to derive corresponding optimality conditions for MPEC.

The rest of the paper is organized as follows. In section 2 we review the theory of 2regularity (for smooth equality constraints) and show, by means of a simple example, that it is relevant in the context of MPEC. Section 3 contains our necessary and sufficient conditions for tangency and a demonstration that this description is always valid under the piecewise MFCQ and can also be useful when the latter condition does not hold. A special form of primal-dual necessary optimality conditions is derived in section 4 . This section also contains a comparison with some alternative approaches to MPEC constraint qualifications and optimality conditions.

We next briefly describe our notation. All vectors will be column-vectors. When writing a pair $(x, y)$, we mean a column-vector composed of two vectors $x$ and $y$. 
For a vector $v$ of arbitrary (finite) dimension, $v_{i}$ will denote its $i$ th component, and for a matrix $M, M_{i}$ will denote its $i$ th row. By $M^{T}$ we shall denote the transposed matrix of $M$. For a differentiable scalar function $\varphi: \Re^{n} \times \Re^{m} \rightarrow \Re, \nabla \varphi(z)$ stands for the row-vector of its partial derivatives at $z=(x, y) \in \Re^{n} \times \Re^{m}$ with respect to all the variables, while $\nabla_{x} \varphi(z)$ will denote the vector of partial derivatives with respect to $x \in \Re^{n}$ (similarly for $y$ ). If $\varphi$ is twice differentiable, $\nabla^{2} \varphi(z)$ denotes its Hessian matrix, and $\nabla_{x}^{2} \varphi(z)$ its Hessian matrix with respect to $x$. For a differentiable vectorfunction $F: \Re^{l} \rightarrow \Re^{k}, F^{\prime}(z)$ will denote its Jacobian, i.e., the $k \times l$ matrix whose $i$ th row is $\nabla F_{i}(z)$. If $F$ is twice differentiable, then $F^{\prime \prime}(z)[h]$ denotes the $k \times l$ matrix whose $i$ th row is $\nabla^{2} F_{i}(z) h, i=1, \ldots, k$. Furthermore, $F^{\prime \prime}(z)[h, p]:=\left(F^{\prime \prime}(z)[h]\right) p$, and $F^{\prime \prime}(z)[h]^{2}:=F^{\prime \prime}(z)[h, h]$. For a linear operator $A: S_{1} \rightarrow S_{2}$, Ker $A=\left\{w \in S_{1} \mid\right.$ $A w=0\}$ is its null space, and $\operatorname{Im} A=\left\{v \in S_{2} \mid v=A w\right.$ for some $\left.w \in S_{1}\right\}$ is its image space. Given a subspace $S$ of an arbitrary space, we shall denote its orthogonal complement in this space by $S^{\perp}$. If $K$ is a cone, then $K^{*}=\{p \mid\langle p, h\rangle \geq 0 \forall h \in K\}$ is the (positive) dual cone of $K$. For a set $K, \mathrm{cl} K$ will stand for its closure. Finally, for a (finite) index set $I$ its cardinality will be denoted by $|I|$.

2. Elements of the theory of 2-regularity. In this section, we describe some results from the theory of 2-regularity for smooth nonlinear mappings [12, 19, 1, 9, 5, $6,7]$. Our emphasis will be on only those constructions that will be used later in the paper. We therefore shall limit our presentation to equality constraints only, to the finite-dimensional setting, and to the case of mappings differentiable at least twice. After introducing the necessary objects, we provide an example which illustrates certain 2-regularity properties of MPEC.

Let $F: \Re^{l} \rightarrow \Re^{k}$ be differentiable at a point $z^{*} \in C$, where

$$
C:=\left\{z \in \Re^{l} \mid F(z)=0\right\} .
$$

We are interested in the tangent cone to the set $C$ at the point $z^{*} \in C$, denoted $T_{C}\left(z^{*}\right)$, which is the set of all vectors $h \in \Re^{l}$ for which there exists a mapping $r: \Re_{+} \rightarrow \Re^{l}$ such that

$$
z^{*}+t h+r(t) \in C \quad \forall t \in \Re_{+}, \quad\|r(t)\|=o(t) .
$$

We note that in the context of constructive constraint qualifications there is no distinction between this definition of the tangent cone and the more general Bouligand tangent cone. In the following, we shall use the given definition, as it is more convenient for our development (and leads to the same results as when using the Bouligand cone anyway). It is well known that

$$
T_{C}\left(z^{*}\right) \subset H_{1}:=\operatorname{Ker} F^{\prime}\left(z^{*}\right),
$$

which is the (first-order) necessary condition of tangency. A sufficient condition is given by the classical Lyusternik theorem: the equality

$$
T_{C}\left(z^{*}\right)=H_{1}
$$

holds if $F$ is (first-order) regular, i.e.,

$$
\operatorname{Im} F^{\prime}\left(z^{*}\right)=\Re^{k} .
$$

In the irregular case when $\operatorname{Im} F^{\prime}\left(z^{*}\right) \neq \Re^{k}$, cone $T_{C}\left(z^{*}\right)$ can be smaller than $H_{1}$. In that case, a more accurate representation is needed. 
Suppose now that $F$ is twice differentiable at $z^{*}$. Then it can be verified that

$$
T_{C}\left(z^{*}\right) \subset H_{2}:=H_{1} \cap\left\{h \mid F^{\prime \prime}\left(z^{*}\right)[h]^{2} \in \operatorname{Im} F^{\prime}\left(z^{*}\right)\right\},
$$

which is the second-order necessary condition of tangency. Let $P$ be the orthogonal projector onto $\left(\operatorname{Im} F^{\prime}\left(z^{*}\right)\right)^{\perp}$ in $\Re^{k}$. With this notation, we can equivalently write

$$
H_{2}=\operatorname{Ker} F^{\prime}\left(z^{*}\right) \cap\left\{h \mid P F^{\prime \prime}\left(z^{*}\right)[h]^{2}=0\right\} .
$$

DeFinition 2.1. Under the assumptions above, the mapping $F$ is called 2-regular at the point $z^{*}$ with respect to an element $h \in \Re^{l}$ if

$$
\operatorname{Im}\left(F^{\prime}\left(z^{*}\right)+P F^{\prime \prime}\left(z^{*}\right)[h]\right)=\Re^{k} .
$$

Obviously, if $F$ is (first-order) regular in the classical sense, i.e., $\operatorname{Im} F^{\prime}\left(z^{*}\right)=\Re^{k}$, then it is 2-regular with respect to every $h$. The following generalization of the classical Lyusternik theorem can be found in $[12,19,1,9,5]$, and more general results under weaker smoothness assumptions, with applications, in $[6,7]$.

Theorem 2.2. Let $F: \Re^{l} \rightarrow \Re^{k}$ be twice differentiable at a point $z^{*} \in \Re^{l}$ such that $F\left(z^{*}\right)=0$. Assume further that $F$ is 2-regular at $z^{*}$ with respect to an element $h \in H_{2}$. Then there exists a mapping $r: \Re_{+} \rightarrow \Re^{l}$ such that

$$
F\left(z^{*}+t h+r(t)\right)=0 \quad \forall t \in \Re_{+}, \quad \text { where }\|r(t)\|=o(t) .
$$

In other words, 2-regularity of $F$ on some $h$ satisfying the second-order necessary conditions of tangency $\left(h \in \mathrm{H}_{2}\right)$ guarantees that this $h$ is indeed a tangent direction. In particular, if $F$ is 2-regular with respect to every $h \in H_{2} \backslash\{0\}$, then

$$
T_{C}\left(z^{*}\right)=H_{2} .
$$

In fact, due to the closedness of the tangent cone, for the above equality to hold it is enough to require that $F$ be 2-regular with respect to every $h$ in some dense subset of $H_{2}$ (i.e., $h \in K$ such that $\operatorname{cl} K=H_{2}$ ).

Note that this representation of the tangent cone subsumes the classical regular case. Indeed, in that case $\operatorname{Im} F^{\prime}\left(z^{*}\right)=\Re^{k}$; hence $\left(\operatorname{Im} F^{\prime}\left(z^{*}\right)\right)^{\perp}=\{0\}, P=0$, and thus $\left\{h \mid P F^{\prime \prime}\left(z^{*}\right)[h]^{2}=0\right\}=\Re^{l}$, and so we have that $T_{C}\left(z^{*}\right)=\operatorname{Ker} F^{\prime}\left(z^{*}\right)=H_{1}=H_{2}$. Of course, the description of tangent directions via constructions of 2-regularity is useful precisely in the absence of classical regularity. It is therefore natural to see what, if anything, the approach of 2-regularity has to offer in the context of MPEC. To quickly convince the reader that 2-regularity is relevant here, we next consider a simple one-dimensional problem and show that sufficient conditions for tangency can be interpreted as 2-regularity of a certain subset of constraints.

Example 2.1. Let $n=m=1$ and consider the set $D$ given by

$$
y \geq 0, \quad g(x, y) \geq 0, \quad 0=F(x, y):=y g(x, y) .
$$

Obviously, the cause of difficulties in MPEC is the degenerate set $I_{0}$, so the case of interest is when $I_{0} \neq \emptyset$. Here, this means that $I_{0}=\{1\}$ while the other index sets introduced in section 1 are empty. Therefore we shall consider a point $\left(x^{*}, y^{*}\right) \in \Re^{2}$ such that

$$
y^{*}=0, \quad g\left(x^{*}, y^{*}\right)=0 .
$$


As is easy to see, in this case

$$
F^{\prime}\left(x^{*}, y^{*}\right)=(0,0)
$$

Hence, the last constraint in (2.2) is not regular (even by itself, separately from the other constraints defining $D$ !). Note further that

$$
F^{\prime \prime}\left(x^{*}, y^{*}\right)=\left(\begin{array}{cc}
0 & \nabla_{x} g\left(x^{*}, y^{*}\right) \\
\nabla_{x} g\left(x^{*}, y^{*}\right) & 2 \nabla_{y} g\left(x^{*}, y^{*}\right)
\end{array}\right)
$$

As is easy to see, the necessary conditions for some $(u, v) \in \Re \times \Re$ to be a tangent direction to $D$ at $\left(x^{*}, y^{*}\right)$ are the following:

$$
\begin{gathered}
v \geq 0, \quad \nabla_{x} g\left(x^{*}, y^{*}\right) u+\nabla_{y} g\left(x^{*}, y^{*}\right) v \geq 0 \\
v\left(\nabla_{x} g\left(x^{*}, y^{*}\right) u+\nabla_{y} g\left(x^{*}, y^{*}\right) v\right)=0 .
\end{gathered}
$$

When are these conditions sufficient? Consider the two possible cases.

Case 1: $v=0$. It is clear that the point $\left(x^{*}+t u, y^{*}+t v\right)=\left(x^{*}+t u, 0\right)$ satisfies the first and third constraints in (2.2) for any $t>0$. Suppose that $\nabla_{x} g\left(x^{*}, y^{*}\right) \neq 0$. Then, by (2.3), if $u \neq 0$, it holds that $\nabla_{x} g\left(x^{*}, y^{*}\right) u>0$. By differentiability of $g$, we then obtain for all $t>0$ small enough that

$$
g\left(x^{*}+t u, 0\right)=t \nabla_{x} g\left(x^{*}, y^{*}\right) u+o(t) \geq 0 .
$$

Hence, $\left(x^{*}+t u, 0\right) \in D \forall t>0$ small, which means that $h=(u, 0) \in T_{D}\left(z^{*}\right)$. Observe now that

$$
F^{\prime}\left(x^{*}, y^{*}\right)+P F^{\prime \prime}\left(x^{*}, y^{*}\right)[h]=\left(0, \nabla_{x} g\left(x^{*}, y^{*}\right) u\right),
$$

and condition $\nabla_{x} g\left(x^{*}, y^{*}\right) \neq 0$ means precisely 2-regularity of $F$ with respect to $h=(u, 0)$, where $u \neq 0$.

Case 2: $v>0$. By $(2.3),\left\langle\nabla g\left(x^{*}, y^{*}\right),(u, v)\right\rangle=0$. Suppose that $\nabla g\left(x^{*}, y^{*}\right) \neq 0$. Then $g$ is regular in the classical sense, and the standard Lyusternik theorem implies that $h=(u, v)$ is a tangent direction to the set $\{(x, y) \mid g(x, y)=0\}$ : there exists some mapping $r(\cdot)=\left(r^{1}(\cdot), r^{2}(\cdot)\right)$ such that

$$
g\left(z^{*}+t h+r(t)\right)=0, \quad\|r(t)\|=o(t) .
$$

Furthermore, for any $t>0$ sufficiently small

$$
y^{*}+t v+r^{1}(t)=t v+o(t) \geq 0 .
$$

Hence, the point $z^{*}+t h+r(t)$ satisfies all constraints in (2.2). Therefore, $h \in T_{D}\left(z^{*}\right)$. Again observe that

$$
\begin{aligned}
F^{\prime}\left(x^{*}, y^{*}\right)+P F^{\prime \prime}\left(x^{*}, y^{*}\right)[h] & =\left(\nabla_{x} g\left(x^{*}, y^{*}\right) v, \nabla_{x} g\left(x^{*}, y^{*}\right) u+2 \nabla_{y} g\left(x^{*}, y^{*}\right) v\right) \\
& =\left(\nabla_{x} g\left(x^{*}, y^{*}\right) v, \nabla_{y} g\left(x^{*}, y^{*}\right) v\right)
\end{aligned}
$$

As is now easy to see, condition $\nabla g\left(x^{*}, y^{*}\right) \neq 0$ is precisely 2-regularity of $F$ with respect to $h=(u, v)$, where $v \neq 0$. (Note that if $\nabla_{x} g\left(x^{*}, y^{*}\right)=0$, then necessarily $\nabla_{y} g\left(x^{*}, y^{*}\right)=0$ (by $(2.3)$, because $v>0$ ). Hence, under our assumption, case $v>0$ can occur only if $\nabla_{x} g\left(x^{*}, y^{*}\right) \neq 0$.) 
We have demonstrated that in both cases the assumption which makes necessary conditions of tangency for some direction $h$ to be sufficient can be interpreted as 2-regularity of the mapping $F$ with respect to this $h$.

In the next section we show that the intuition derived from this simple onedimensional case is essentially valid in general. Specifically, 2-regularity of constraints that have to be satisfied as equalities (in a neighborhood of the point being considered) yields a precise description of the tangent cone to complementarity constraints at this point.

3. 2-regularity for MPEC. We start with recalling some useful objects from the MPEC theory $[11,10,18,15]$. The standard necessary condition of tangency in MPEC is

$$
T_{D}\left(z^{*}\right) \subset L
$$

where $L$ is the "linearized cone":

$$
L:=\left\{\begin{array}{l|l}
(u, v) \in \Re^{n} \times \Re^{m} & \begin{array}{l}
\left\langle\nabla g_{i}\left(z^{*}\right),(u, v)\right\rangle \geq 0, v_{i} \geq 0, \quad i \in I_{0}, \\
v_{i}\left\langle\nabla g_{i}\left(z^{*}\right),(u, v)\right\rangle=0, \quad i \in I_{0}, \\
\left\langle\nabla g_{i}\left(z^{*}\right),(u, v)\right\rangle=0, \quad i \in I_{g}
\end{array}
\end{array}\right\} .
$$

Linearization here is understood differently from the usual notion in nonlinear programming. Indeed, $L$ is not a polyhedral cone, except when the degenerate set $I_{0}$ is empty. Instead, $L$ consists of a finite union of polyhedral cones. It is known [11] that (3.1) holds as equality when $g(\cdot, \cdot)$ is an affine function or when $z^{*} \in D$ is (strongly) regular in the sense of [17].

It is also clear that $T_{D}\left(z^{*}\right)=L$ under some piecewise constraint qualification. Let $(R, Q)$ be the family of partitions of the degenerate set $I_{0}$, i.e., $R \cup Q=I_{0}, R \cap Q=\emptyset$. Associated with each partition $(R, Q)$, define the branch of the feasible set

$$
D_{R Q}:=\left\{\begin{array}{l|l}
(x, y) & \begin{array}{l}
g_{i}(x, y) \geq 0, y_{i}=0, \quad i \in R \\
g_{i}(x, y)=0, y_{i} \geq 0, \quad i \in Q \cup I_{g}
\end{array}
\end{array}\right\} .
$$

It is easy to see that

$$
\bigcup_{(R, Q)}\left(D_{R Q} \cap \mathcal{V}\right)=D \cap \mathcal{V}, \quad T_{D}\left(z^{*}\right)=\bigcup_{(R, Q)} T_{D_{R Q}}\left(z^{*}\right)
$$

where $\mathcal{V}$ is a neighborhood of $z^{*}$. If one assumes that, say, the MFCQ is satisfied at $z^{*}$ for constraints defining each of the sets $D_{R Q}$, then the corresponding branch of $L$ given by

$$
L_{R Q}:=\left\{\begin{array}{l|l}
(u, v) & \begin{array}{l}
v_{i}=0,\left\langle\nabla g_{i}\left(z^{*}\right),(u, v)\right\rangle \geq 0, \quad i \in R, \\
\left\langle\nabla g_{i}\left(z^{*}\right),(u, v)\right\rangle=0, \quad i \in Q \cup I_{g}, \\
v_{i} \geq 0, \quad i \in Q,
\end{array}
\end{array}\right\}
$$

will represent necessary and sufficient conditions of tangency for this $D_{R Q}$. Putting together all the pieces, one then obtains that $T_{D}\left(z^{*}\right)=L$.

In general, however, (3.1) does not hold as equality, and so further analysis is needed to obtain a precise description of the tangent directions. We next show how this can be done with the help of 2-regularity outlined in section 2 . 
Recall the mapping $F: \Re^{n} \times \Re^{m} \rightarrow \Re^{\left|I_{0}\right|+\left|I_{g}\right|}$ associated with equality constraints in $D^{*}$ (which is locally equivalent to $D$ ):

$$
F_{i}(x, y):= \begin{cases}y_{i} g_{i}(x, y), & i \in I_{0} \\ g_{i}(x, y), & i \in I_{g}\end{cases}
$$

With this notation,

$$
D^{*}:=\left\{\begin{array}{l|l}
(x, y) & \begin{array}{l}
y_{i} \geq 0, g_{i}(x, y) \geq 0, \quad i \in I_{0} \\
F(x, y)=0
\end{array}
\end{array}\right\}
$$

Note that the $i$ th row of $F^{\prime}\left(x^{*}, y^{*}\right)$ is

$$
F^{\prime}\left(x^{*}, y^{*}\right)_{i}=\nabla F_{i}\left(x^{*}, y^{*}\right)= \begin{cases}0, & i \in I_{0} \\ \nabla g_{i}\left(x^{*}, y^{*}\right), & i \in I_{g}\end{cases}
$$

Observe that $F$ cannot be regular unless the degenerate set $I_{0}$ is empty. On the other hand, it is quite natural to study $F$ from the point of view of 2-regularity. Note, however, that $F$ represents only part of the constraints. Indeed, application of 2-regularity (and arguably, of any general regularity concept) to MPEC cannot be straightforward. This can already be seen from the one-dimensional example considered in section 2. The current general theory for irregular problems does not permit mixed equality and inequality constraints, except in some very special cases. Within the general theory available, if one is to insist on mixed constraints, then all irregularity has to be induced by equalities with inequalities being regular [2,9], or vice versa [8]. Yet it can be verified that for MPEC even these two extreme cases cannot be applied. Fortunately, and thanks to the special complementarity structure of $D$, it appears possible to develop a special approach for MPEC, different from the general theory. We next show that whenever tangent directions can be characterized via 2regularity for the subset of constraints in $D^{*}$ corresponding to $F(x, y)=0$, then these directions are automatically tangent for the complete set of constraints, without any further assumptions involving these constraints.

We start by computing some objects described in section 2. Let $A_{g}$ be the $\left|I_{g}\right| \times$ $(n+m)$ matrix with rows $\nabla g_{i}\left(z^{*}\right), i \in I_{g}$. With this notation,

$$
\operatorname{Ker} F^{\prime}\left(z^{*}\right)=\operatorname{Ker} A_{g}
$$

Furthermore,

$$
\begin{gathered}
\operatorname{Im} F^{\prime}\left(z^{*}\right)=\left\{(0, w) \in \Re^{\left|I_{0}\right|} \times \Re^{\left|I_{g}\right|} \mid w \in \operatorname{Im} A_{g}\right\}, \\
\left(\operatorname{Im} F^{\prime}\left(z^{*}\right)\right)^{\perp}=\left\{(p, q) \in \Re^{\left|I_{0}\right|} \times \Re^{\left|I_{g}\right|} \mid q \in\left(\operatorname{Im} A_{g}\right)^{\perp}\right\} .
\end{gathered}
$$

Now, let $P$ be the orthogonal projector onto $\left(\operatorname{Im} F^{\prime}\left(z^{*}\right)\right)^{\perp}$. Then

$$
P(x, y)=\left(x, P_{g} y\right)
$$

where $P_{g}$ is the orthogonal projector onto $\left(\operatorname{Im} A_{g}\right)^{\perp}$. Differentiating $F$ twice, it can be verified that for any $(u, v) \in \Re^{n} \times \Re^{m}$,

$$
F^{\prime \prime}\left(z^{*}\right)[(u, v)]=\left(\begin{array}{c}
B_{0}[(u, v)] \\
B_{g}[(u, v)]
\end{array}\right)
$$


where $B_{0}[(u, v)]$ is the $\left|I_{0}\right| \times(n+m)$ matrix with rows

$$
B_{0}[(u, v)]_{i}=v_{i} \nabla g_{i}\left(z^{*}\right)+\left\langle\nabla g_{i}\left(z^{*}\right),(u, v)\right\rangle e^{i}, \quad i \in I_{0},
$$

with $e^{i} \in \Re^{n+m}$ being the vector of zeros except for the $(n+i)$ th component, which is equal to one; and $B_{g}[(u, v)]$ is the $\left|I_{g}\right| \times(n+m)$ matrix with rows

$$
B_{g}[(u, v)]_{i}=\nabla^{2} g_{i}\left(z^{*}\right)(u, v), \quad i \in I_{g} .
$$

Observe further that

$$
\left(B_{0}[(u, v)]^{2}\right)_{i}=2 v_{i}\left\langle\nabla g_{i}\left(z^{*}\right),(u, v)\right\rangle .
$$

Taking into account this information, we have that

$$
P F^{\prime \prime}\left(z^{*}\right)[(u, v)]^{2}=0 \quad \Leftrightarrow \quad B_{0}[(u, v)]^{2}=0, P_{g} B_{g}[(u, v)]^{2}=0,
$$

which in turn is equivalent to

$$
v_{i}\left\langle\nabla g_{i}\left(z^{*}\right),(u, v)\right\rangle=0, \quad i \in I_{0}, \quad P_{g} B_{g}[(u, v)]^{2}=0 .
$$

We can now state necessary conditions for tangent directions to equality constraints in $D^{*}$, in terms of cone $H_{2}$ defined by (2.1):

$$
\begin{aligned}
& \left\langle\nabla g_{i}\left(z^{*}\right),(u, v)\right\rangle=0, \quad i \in I_{g}, \\
& v_{i}\left\langle\nabla g_{i}\left(z^{*}\right),(u, v)\right\rangle=0, \quad i \in I_{0}, \\
& P_{g} B_{g}[(u, v)]^{2}=0 .
\end{aligned}
$$

Putting these together with standard necessary conditions for inequality constraints in $D^{*}$, we obtain

$$
T_{D}\left(z^{*}\right) \subset H
$$

where

$$
H:=\left\{\begin{array}{l|l}
(u, v) \mid \begin{array}{l}
\left\langle\nabla g_{i}\left(z^{*}\right),(u, v)\right\rangle \geq 0, v_{i} \geq 0, \quad i \in I_{0}, \\
v_{i}\left\langle\nabla g_{i}\left(z^{*}\right),(u, v)\right\rangle=0, \quad i \in I_{0}, \\
\left\langle\nabla g_{i}\left(z^{*}\right),(u, v)\right\rangle=0, \quad i \in I_{g}, \\
P_{g} B_{g}[(u, v)]^{2}=0
\end{array}
\end{array}\right\}
$$

Note that the above is not a standard set of necessary conditions: we have a mix of second-order conditions for equality constraints with first-order conditions for inequality constraints. In general, $H$ is not piecewise polyhedral, although it can be such under some natural assumptions discussed below. But perhaps more interesting is the difficult case when $H$ is not piecewise polyhedral. Even in that case, as we shall see, (3.10) gives a precise (i.e., $T_{D}\left(z^{*}\right)=H$ ) constructive description of tangency whenever the mapping $F$ is 2-regular. This can further be used for deriving a special form of primal-dual optimality conditions in the situation where other techniques are not applicable.

We are now ready to state our description of the tangent cone to complementarity constraints.

TheOREM 3.1. Let $g$ be twice differentiable at $z^{*} \in D$, where $D$ is given by (1.1). Then the following statements hold. 
1. If $h \in T_{D}\left(z^{*}\right)$, then $h \in H$.

2. If $F$ defined by (3.6) is 2-regular at $z^{*}$ with respect to $h \in H$, then $h \in T_{D}\left(z^{*}\right)$.

Proof. In view of the preceding discussion, the first assertion of the theorem requires no further justification.

Suppose now that $F$ is 2-regular with respect to some $h=(u, v) \in H$. By Theorem 2.2, it then follows that $h$ is tangent to the set $\{z \mid F(z)=0\}$ :

$$
F\left(z^{*}+t h+r(t)\right)=0, \quad\|r(t)\|=o(t),
$$

where $r(\cdot)=\left(r^{1}(\cdot), r^{2}(\cdot)\right)$ is the mapping from Theorem 2.2. By the definition of $F$, this is equivalent to

$$
\begin{aligned}
\left(y_{i}^{*}+t v_{i}+r_{i}^{2}(t)\right) g_{i}\left(x^{*}+t u+r^{1}(t), y^{*}+t v+r^{2}(t)\right) & =0, \quad i \in I_{0}, \\
g_{i}\left(x^{*}+t v+r^{1}(t), y^{*}+t v+r^{2}(t)\right) & =0, \quad i \in I_{g} .
\end{aligned}
$$

To prove that $h \in T_{D}\left(z^{*}\right)$, it remains to show that $z^{*}+t h+r(t)$ satisfies the remaining inequality constraints (for $t>0$ sufficiently small). According to prior calculations,

$$
F^{\prime}\left(z^{*}\right)+P F^{\prime \prime}\left(z^{*}\right)[(u, v)]=\left(\begin{array}{c}
B_{0}[(u, v)] \\
A_{g}+P_{g} B_{g}[(u, v)]
\end{array}\right),
$$

and the assumption of 2-regularity means that the matrix on the right-hand side has full row rank. In particular, $B_{0}[(u, v)]$ must have full row rank. Hence (see (3.7)),

$$
v_{i} \nabla g_{i}\left(z^{*}\right)+\left\langle\nabla g_{i}\left(z^{*}\right),(u, v)\right\rangle e^{i}, i \in I_{0}, \text { are linearly independent. }
$$

Define

$$
I_{0}^{0}:=\left\{i \in I_{0} \mid v_{i}=0\right\}, \quad I_{0}^{1}:=I_{0} \backslash I_{0}^{0}=\left\{i \in I_{0} \mid v_{i}>0\right\} .
$$

It immediately follows from (3.10) that

$$
\left\langle\nabla g_{i}\left(z^{*}\right),(u, v)\right\rangle=0, \quad i \in I_{0}^{1} .
$$

Note that the linear independence of vectors in (3.12) corresponding to $i \in I_{0}^{0}$ means that $\left\langle\nabla g_{i}\left(z^{*}\right),(u, v)\right\rangle \neq 0, i \in I_{0}^{0}$. Further, taking into account (3.10), this is equivalent to

$$
\left\langle\nabla g_{i}\left(z^{*}\right),(u, v)\right\rangle>0, \quad i \in I_{0}^{0} .
$$

Using (3.13), differentiability of $g$ implies that for all $t>0$ sufficiently small

$$
g_{i}\left(x^{*}+t v+r^{1}(t), y^{*}+t v+r^{2}(t)\right)=t\left\langle\nabla g_{i}\left(z^{*}\right),(u, v)\right\rangle+o(t)>0, \quad i \in I_{0}^{0} .
$$

The latter relation, together with (3.11), yields

$$
y_{i}^{*}+t v_{i}+r_{i}^{2}(t)=r_{i}^{2}(t)=0, \quad i \in I_{0}^{0} .
$$

Similarly, for all $t>0$ small enough,

$$
y_{i}^{*}+t v_{i}+r_{i}^{2}(t)=t v_{i}+r_{i}^{2}(t)>0, \quad i \in I_{0}^{1},
$$

and so (3.11) implies that

$$
g_{i}\left(x^{*}+t v+r^{1}(t), y^{*}+t v+r^{2}(t)\right)=0, \quad i \in I_{0}^{1} .
$$

In particular, we have established that for all $t>0$ sufficiently small, $(x, y)=z^{*}+$ $t h+r(t)$ satisfies the inequality constraints $y_{i} \geq 0, g_{i}(x, y) \geq 0, i \in I_{0}=I_{0}^{0} \cup I_{0}^{1}$, which completes the proof that $h \in T_{D}\left(z^{*}\right)$. 
COROLlaRY 3.2. The following statements hold.

1. If there exists some set $K \subset H$ such that $F$ is 2-regular with respect to every $h \in K$ and $\operatorname{cl} K=H$, then $T_{D}\left(z^{*}\right)=H$.

2. If

$$
\nabla g_{i}\left(z^{*}\right), \quad i \in I_{g}, \quad \text { are linearly independent, }
$$

then $H=L$. In particular, under the assumption of the previous item, $T_{D}\left(z^{*}\right)=L$.

Proof. The first assertion follows from Theorem 3.1 and closedness of the tangent cone. For the second assertion, just notice that (3.14) implies that $\left(\operatorname{Im} A_{g}\right)^{\perp}=\{0\}$, and so $P_{g}=0$. Hence, $H=L$.

Therefore, whenever there exists a dense subset of $H$ with respect to which $F$ is 2-regular, $H$ gives a precise description of the tangent cone. If (3.14) also holds, necessary conditions for tangency take a simpler piecewise polyhedral form.

We next show that the piecewise MFCQ is a sufficient condition for 2-regularity of $F$ on a certain dense subset of $H$, as well as for (3.14). A branch $D_{R Q}$, defined by (3.3), satisfies MFCQ if

$$
\begin{aligned}
& \nabla g_{i}\left(z^{*}\right), \quad i \in Q \cup I_{g}, \quad e^{i}, i \in R, \quad \text { are linearly independent, and } \\
& \exists(u, v) \in \Re^{n} \times \Re^{m} \text { such that } \\
& v_{i}=0, \quad\left\langle\nabla g_{i}\left(z^{*}\right),(u, v)\right\rangle>0, \quad i \in R, \\
& \left\langle\nabla g_{i}\left(z^{*}\right),(u, v)\right\rangle=0, \quad i \in Q \cup I_{g}, \\
& v_{i}>0, \quad i \in Q,
\end{aligned}
$$

where $e^{i}=(0,0, \ldots, 1, \ldots, 0) \in \Re^{n+m}$ with one in the $(n+i)$ th position. Under the piecewise MFCQ, for each branch $D_{R Q}$ the (classical) linearized cone $L_{R Q}$, defined by (3.5), represents all the tangent directions. Note that, as discussed above, the fact that a piecewise constraint qualification implies the equality $T_{D}\left(z^{*}\right)=L$ is fairly obvious. The significance of the following result lies not in establishing this equality but in providing a new interpretation for piecewise MFCQ in terms of 2-regularity of a subset of constraints of the original nondecomposed problem. Since piecewise MFCQ is arguably the most natural condition guaranteeing that $T_{D}\left(z^{*}\right)=L$, this goes to show that the introduced 2-regularity condition is also natural for MPEC. Furthermore, piecewise MFCQ is merely a sufficient condition for 2-regularity, as would be demonstrated by an example.

TheOrem 3.3. Suppose that piecewise MFCQ holds at $z^{*} \in D$. Then $F$ is 2regular with respect to every $h=(u, v) \in \Re^{n} \times \Re^{m}$ satisfying (3.15) for some pair $(R, Q)$, and it holds that $T_{D}\left(z^{*}\right)=H=L$.

Proof. Consider an arbitrary branch $D_{R Q}$ of the feasible set $D$, and an arbitrary $h=(u, v)$ satisfying (3.15). We have to show that the rows of matrix

$$
F^{\prime}\left(z^{*}\right)+P F^{\prime \prime}\left(z^{*}\right)[(u, v)]=\left(\begin{array}{c}
B_{0}[(u, v)] \\
A_{g}+P_{g} B_{g}[(u, v)]
\end{array}\right)=\left(\begin{array}{c}
B_{0}[(u, v)] \\
A_{g}
\end{array}\right)
$$

are linearly independent. By (3.7) and (3.15), we have that

$$
B_{0}[(u, v)]_{i}= \begin{cases}\left\langle\nabla g_{i}\left(z^{*}\right),(u, v)\right\rangle e^{i}, & i \in R \\ v_{i} \nabla g_{i}\left(z^{*}\right), & i \in Q\end{cases}
$$

By (3.15), it further holds that

$$
\begin{aligned}
& \left\langle\nabla g_{i}\left(z^{*}\right),(u, v)\right\rangle>0, \quad i \in R, \\
& v_{i}>0, \quad i \in Q .
\end{aligned}
$$


The linear independence condition in (3.15) now implies the desired linear independence of the rows of $F^{\prime}\left(z^{*}\right)+P F^{\prime \prime}\left(z^{*}\right)[(u, v)]$.

Taking into account that MFCQ subsumes (3.14), the last assertion follows immediately from Corollary 3.2.

The following example demonstrates that 2-regularity of $F$ is a weaker condition than piecewise MFCQ, i.e., the former may be satisfied when the latter is not. Furthermore, it shows that our results can yield a relatively simple description of the tangent cone even in the difficult situation when piecewise constraint qualifications fail.

Example 3.1. Let $n=1, m=2$, and consider the set $D$ given by (1.1), where

$$
g_{1}(x, y)=x+y_{1}, \quad g_{2}(x, y)=x^{2}-\left(y_{2}-a\right)^{2},
$$

with $a>0$ being any given number. Consider the point $z^{*}=\left(x^{*}, y^{*}\right)$ with $x^{*}=0$, $y_{1}^{*}=0, y_{2}^{*}=a$. We have that $z^{*} \in D$ and $I_{0}=\{1\}, I_{g}=\{2\}$,

$$
H=\left\{\begin{array}{l|l}
(u, v) & \begin{array}{l}
v_{1} \geq 0, u+v_{1} \geq 0 \\
v_{1}\left(u+v_{1}\right)=0 \\
u^{2}-v_{2}^{2}=0
\end{array}
\end{array}\right\} .
$$

Since $\nabla g_{2}\left(z^{*}\right)=0$, it is clear from (3.15) that the piecewise MFCQ does not hold at $z^{*}$. By direct computations, we have that

$$
F^{\prime}\left(z^{*}\right)+P F^{\prime \prime}\left(z^{*}\right)[(u, v)]=\left(\begin{array}{ccc}
v_{1} & u+2 v_{1} & 0 \\
2 u & 0 & 2 v_{2}
\end{array}\right) .
$$

It can be easily seen that $F$ is 2-regular with respect to any element in $H \backslash\{0\}$, and hence, $T_{D}\left(z^{*}\right)=H$.

It is further interesting to note that even though $H \neq L$, the structure of $H$ is no more complex than that of $L$. Indeed, observe that $u^{2}-v_{2}^{2}=0 \Leftrightarrow u=v_{2}$ or $u=-v_{2}$. Hence, cone $H$ is piecewise polyhedral, just like $L$. However, $H=T_{D}\left(z^{*}\right) \neq L$. This shows that 2-regularity can give a constructive description of the tangent cone when $T_{D}\left(z^{*}\right) \neq L$, even without going beyond the situation where the tangent cone is piecewise polyhedral.

We conclude this example by noting that it remains valid if we perturb $g$ by any function $\delta$ such that $\delta(z)=o\left(\left\|z-z^{*}\right\|^{2}\right)$. In particular, this would not affect 2-regularity properties of $F$ or constraints defining cone $H$ (this is because the first and second derivatives at $z^{*}$ would not change). After such a perturbation, it would still hold that $H=T_{D}\left(z^{*}\right) \neq L$.

4. Optimality conditions. In this section we show that 2-regularity can be used to derive optimality conditions, including a special form of primal-dual conditions, in situations when alternative approaches may not be applicable. Our results are meant as a complement to (rather than a substitute for) other types of optimality conditions for MPEC, some of which we shall discuss below.

We start with the primal form of optimality conditions, which can be derived in a standard way using the relations

$$
\operatorname{cl} K \subset T_{D}\left(x^{*}\right) \subset H,
$$

where

$$
K:=\{h \in H \mid F \text { is 2-regular with respect to } h\} .
$$


THEOREM 4.1. Let $f$ be once differentiable and $g$ be twice differentiable at a point $z^{*} \in D$.

If $z^{*}$ is a local solution of (1.2), then

$$
\left\langle\nabla f\left(z^{*}\right), h\right\rangle \geq 0 \quad \forall h \in \operatorname{cl} K .
$$

If it holds that

$$
\left\langle\nabla f\left(z^{*}\right), h\right\rangle>0 \quad \forall h \in H \backslash\{0\},
$$

then $z^{*}$ is an isolated local solution of (1.2).

Note that when $\mathrm{cl} K=H=L$ (e.g., under the assumptions of Corollary 3.2), we recover $B$-stationarity as defined in [18], which is generally considered one of the natural primal stationarity concepts for MPEC (see also Example 4.1).

To obtain a primal-dual form of optimality conditions, one way is to compute the dual cone of $K$. In general, this is a difficult problem, as this cone need not be even piecewise polyhedral. However, we are able to derive special primal-dual optimality conditions using a different technique. This is possible under the assumption that there exists some $\bar{h} \in K$ for which inequality in (4.1) holds as equality (a "critical" direction). This assumption is justifiable in this context, and it is quite common in the literature, e.g., $[3,16]$. If $\left\langle\nabla f\left(z^{*}\right), \bar{h}\right\rangle>0$ for a given $\bar{h} \in K$, then it is clear that the same property holds for all $h$ in some neighborhood $\mathcal{U}$ of $\bar{h}$. Hence, on $\mathcal{U}$ there is no contradiction with necessary optimality conditions, and $z^{*}$ is still a candidate for being a solution. There is no more local information to be extracted using this $\bar{h}$. On the other hand, if $\left\langle\nabla f\left(z^{*}\right), \bar{h}\right\rangle=0$, a further investigation is needed, and indeed more local information relevant to optimality of $z^{*}$ can be obtained. Note that in the following result, 2-regularity of $F$ is assumed with respect to this $\bar{h}$ only, and in particular, the knowledge of the entire tangent cone is not necessary. We first state our optimality conditions using the same objects as in 2-regularity constructions in section 3, which allows a more compact form. After the proof, we shall rewrite them in terms of the original problem data and give comparisons with some other results in the literature.

THEOREM 4.2. Let $f$ be once and $g$ be twice differentiable at a point $z^{*} \in D$, which is a local minimizer for problem (1.2). Assume further that

$$
\exists \bar{h} \in K \text { such that }\left\langle\nabla f\left(z^{*}\right), \bar{h}\right\rangle=0 .
$$

Then there exist unique $\bar{\lambda} \in \Re^{\left|I_{0}\right|}$ and $\bar{\mu}^{1}, \bar{\mu}^{2} \in \Re^{\left|I_{g}\right|}$ (all depending on $\bar{h}$ ) such that

$$
\begin{gathered}
\nabla f\left(z^{*}\right)+\left(B_{0}[\bar{h}]\right)^{T} \bar{\lambda}+A_{g}^{T} \bar{\mu}^{1}+\left(B_{g}[\bar{h}]\right)^{T} \bar{\mu}^{2}=0, \\
\bar{\mu}^{1} \in \operatorname{Im} A_{g}, \quad \bar{\mu}^{2} \in\left(\operatorname{Im} A_{g}\right)^{\perp},
\end{gathered}
$$

where the index sets $I_{0}$ and $I_{g}$ are as defined earlier.

Moreover, the multiplier $\bar{\mu}^{2}$ satisfies the following additional condition: for every $h$ such that

$$
h \in \operatorname{Ker} A_{g}, \quad B_{0}[\bar{h}, h]=0, \quad B_{g}[\bar{h}, h] \in \operatorname{Im} A_{g},
$$

it holds that

$$
\left\langle\bar{\mu}^{2}, B_{g}[h]^{2}\right\rangle \geq 0
$$


Proof. It can be easily seen from Definition 2.1 that if 2-regularity of $F$ holds for some $\bar{h}=(\bar{u}, \bar{v}) \in H$, then it holds for all $h$ sufficiently close to $\bar{h}$. In other words, there exists a neighborhood $\mathcal{U}$ of $\bar{h}$ in $\Re^{n} \times \Re^{m}$ such that

$$
H \cap \mathcal{U} \subset K .
$$

Hence, by Theorem 4.1, we have that

$$
\left\langle\nabla f\left(z^{*}\right), h\right\rangle \geq 0 \quad \forall h \in H \cap \mathcal{U} .
$$

Observe that the latter relation and (4.3) imply that $\bar{h}$ is a local solution of the optimization problem

$$
\min _{h \in H}\left\langle\nabla f\left(z^{*}\right), h\right\rangle .
$$

The feasible set $H$ of problem (4.8), given by (3.10), can be equivalently written in the following form (recalling definitions of $B_{0}$ and $A_{g}$ ):

$$
H=\left\{\begin{array}{l|l}
h=(u, v) & \begin{array}{l}
v_{i} \geq 0,\left\langle\nabla g_{i}\left(z^{*}\right), h\right\rangle \geq 0, \quad i \in I_{0}, \\
\frac{1}{2} B_{0}[h]^{2}=0, \\
A_{g} h+\frac{1}{2} P_{g} B_{g}[h]^{2}=0
\end{array}
\end{array}\right\} .
$$

Here we have also taken into account the following obvious observation: for any $h$ the elements $A_{g} h$ and $\frac{1}{2} P_{g} B_{g}[h]^{2}$ belong to orthogonal subspaces in $\Re^{\left|I_{g}\right|}$, and hence, they both are equal to zero if and only if their sum is equal to zero.

Next we show that in a neighborhood of $\bar{h}$ the set $H$ is completely defined by equality constraints only, so in our local considerations we can omit the inequality constraints in (4.9). Indeed, recall that since $F$ is 2-regular with respect to $\bar{h}$, we have by (3.13) that

$$
\left\langle\nabla g_{i}\left(z^{*}\right), \bar{h}\right\rangle>0, \quad i \in I_{0}^{0}
$$

where

$$
I_{0}^{0}=\left\{i \in I_{0} \mid \bar{v}_{i}=0\right\} .
$$

Let $h$ satisfy the equality $B_{0}[h]^{2}=0$. By (3.9), we then obtain that

$$
v_{i}\left\langle\nabla g_{i}\left(z^{*}\right), h\right\rangle=0, \quad i \in I_{0} .
$$

This relation and (4.10) imply that if $h=(u, v)$ is sufficiently close to $\bar{h}$, then it must hold that

$$
\begin{aligned}
& v_{i}=0,\left\langle\nabla g_{i}\left(z^{*}\right), h\right\rangle>0, \quad i \in I_{0}^{0}, \\
& v_{i}>0,\left\langle\nabla g_{i}\left(z^{*}\right), h\right\rangle=0, \quad i \in I_{0}^{1},
\end{aligned}
$$

where

$$
I_{0}^{1}=I_{0} \backslash I_{0}^{0}=\left\{i \in I_{0} \mid \bar{v}_{i}>0\right\} .
$$

We have therefore established that there exists a neighborhood $\mathcal{V}$ of $\bar{h}$ such that whenever $h \in \mathcal{V}$ satisfies the equality constraints in (4.9), it also satisfies the inequality constraints (by (4.12)). In other words, $H$ is completely defined by its equality constraints (locally, in $\mathcal{V}$ ). Writing these equality constraints as $\Phi(h)=0$, where

$$
\Phi(h)=\left(\begin{array}{c}
\frac{1}{2} B_{0}[h]^{2} \\
A_{g} h+\frac{1}{2} P_{g} B_{g}[h]^{2}
\end{array}\right),
$$


we conclude that problem (4.8) is locally equivalent to

$$
\min _{\Phi(h)=0}\left\langle\nabla f\left(z^{*}\right), h\right\rangle .
$$

Furthermore,

$$
\Phi^{\prime}(\bar{h})=\left(\begin{array}{c}
B_{0}[\bar{h}] \\
A_{g}+P_{g} B_{g}[\bar{h}]
\end{array}\right),
$$

and, as we have verified in the proof of Theorem 3.1, the assumption of 2-regularity of $F$ with respect to $\bar{h}$ means that the matrix on the right-hand side has full row rank. Thus $\Phi$ is regular at $\bar{h}$ in the classical sense. This means that classical firstand second-order optimality conditions for regular equality-constrained problems are applicable for problem (4.14) at its local solution $\bar{h}$. To this end, define the standard Lagrangian for (4.14):

$$
\begin{aligned}
L(h, \lambda, \mu) & =\left\langle\nabla f\left(z^{*}\right), h\right\rangle+\langle(\lambda, \mu), \Phi(h)\rangle \\
& =\left\langle\nabla f\left(z^{*}\right), h\right\rangle+\left\langle\lambda, \frac{1}{2} B_{0}[h]^{2}\right\rangle+\left\langle\mu, A_{g} h+\frac{1}{2} P_{g} B_{g}[h]^{2}\right\rangle .
\end{aligned}
$$

Representing $\mu$ as $\mu=\mu^{1}+\mu^{2}$, where $\mu^{1} \in \operatorname{Im} A_{g}$ and $\mu^{2} \in\left(\operatorname{Im} A_{g}\right)^{\perp}$, the Lagrangian can be further rewritten as

$$
L(h, \lambda, \mu)=\left\langle\nabla f\left(z^{*}\right), h\right\rangle+\frac{1}{2}\left\langle\lambda, B_{0}[h]^{2}\right\rangle+\left\langle\mu^{1}, A_{g} h\right\rangle+\frac{1}{2}\left\langle\mu^{2}, B_{g}[h]^{2}\right\rangle .
$$

Now, by the classical first-order necessary optimality conditions, there exists a (unique, by necessity) pair $(\bar{\lambda}, \bar{\mu}) \in \Re^{\left|I_{0}\right|} \times \Re^{\left|I_{g}\right|}$ such that

$$
\nabla_{h} L(\bar{h}, \bar{\lambda}, \bar{\mu})=0
$$

and, by the second-order necessary optimality conditions, it further holds that

$$
\left\langle\nabla_{h}^{2} L(\bar{h}, \bar{\lambda}, \bar{\mu}) h, h\right\rangle \geq 0 \quad \forall h \in \operatorname{Ker} \Phi^{\prime}(\bar{h}) .
$$

Computing all the derivatives involved (by taking into account (4.15) and (4.16)), we obtain the asserted results.

Using the definition of $A_{g},(3.7),(3.8)$, and (4.12) (for $h=\bar{h}$ ), we can rewrite our optimality conditions explicitly in terms of the problem data. Specifically, for (4.4) and the second relation in (4.5), we obtain

$$
\nabla f\left(z^{*}\right)+\sum_{i \in I_{0}^{0}} \tilde{\lambda}_{i} e^{i}+\sum_{i \in I_{0}^{1}} \tilde{\lambda}_{i} \nabla g_{i}\left(z^{*}\right)+\sum_{i \in I_{g}}\left(\bar{\mu}_{i}^{1} \nabla g_{i}\left(z^{*}\right)+\bar{\mu}_{i}^{2} \nabla^{2} g_{i}\left(z^{*}\right) \bar{h}\right)=0
$$

and

$$
\sum_{i \in I_{g}} \bar{\mu}_{i}^{2} \nabla g_{i}\left(z^{*}\right)=0
$$

where $e^{i}$ is as defined earlier, the index sets $I_{0}^{0}$ and $I_{0}^{1}$ are defined in (4.11) and (4.13), respectively, and

$$
\tilde{\lambda}_{i}= \begin{cases}\bar{\lambda}_{i}\left\langle\nabla g_{i}\left(z^{*}\right), \bar{h}\right\rangle, & i \in I_{0}^{0}, \\ \bar{\lambda}_{i} \bar{v}_{i}, & i \in I_{0}^{1} .\end{cases}
$$


Similarly, we can rewrite the first two conditions on $h=(u, v)$ in (4.6) as follows:

$$
\left\langle\nabla g_{i}\left(z^{*}\right), h\right\rangle=0, \quad i \in I_{g},
$$

and

$$
v_{i}=0, \quad i \in I_{0}^{0}, \quad\left\langle\nabla g_{i}\left(z^{*}\right), h\right\rangle=0, \quad i \in I_{0}^{1},
$$

respectively. Finally, taking into account (3.9), condition (4.7) takes the form

$$
\sum_{i \in I_{g}} \bar{\mu}_{i}^{2}\left\langle\nabla^{2} g_{i}\left(z^{*}\right) h, h\right\rangle \geq 0 .
$$

Note that if the linear independence condition (3.14) is satisfied, then necessarily $\bar{\mu}^{2}=0$, and our optimality conditions take a simpler form. We next comment on some other approaches to constraint qualifications and optimality conditions for MPEC.

First, note that Theorem 4.2 subsumes optimality conditions for the nondegenerate case of $I_{0}=\emptyset$, but in a way different from conditions based on disjunctive programming $[11,10]$. Observe that if $I_{0}=\emptyset$, then $F$ is composed of components $g_{i}, i \in I_{g}$. Further, if $\nabla g_{i}\left(z^{*}\right), i \in I_{g}$, are linearly independent (condition (3.14)), then $F$ is regular at $z^{*}$ in the classical sense and, hence, 2-regular with respect to every $h$. We can then choose $\bar{h}=0$ in (4.3). With this choice, the primal-dual characterization of optimality given by Theorem 4.2 reduces to $\nabla f\left(z^{*}\right)=\sum_{i \in I_{g}} \bar{\mu}_{i}^{1} \nabla g_{i}\left(z^{*}\right)$, which is precisely the Karush-Kuhn-Tucker conditions in the situation where $I_{0}=\emptyset$ and $I_{y}=\emptyset$. (Recall that we set $I_{y}=\emptyset$ merely to simplify notation; if $I_{y} \neq \emptyset$, then the gradients of $y_{i}=0, i \in I_{y}$, enter into play in the obvious way.)

In [15], some conditions are obtained which ensure that the tangent cone and/or its dual can be computed as corresponding cones of certain standard nonlinear programs associated with MPEC. In particular, these cones are therefore polyhedral. Since this need not be the case in our development, it is clear that the two approaches are principally different. The motivation of [15] is to identify situations where MPEC optimality can be characterized without an excessive combinatorial burden of disjunctive programming. Note that our constraint qualification does not require decomposition of the feasible region for its verification. In this sense, it also contributes to the same goal.

We next comment on some results in [18] and, in particular, show that our approach can be useful to verify optimality when [18] may not be applicable, and vice versa. We start with some general comments and then give a number of examples.

For the feasible set $D$ given by (1.1), all constraint qualifications considered in [18] are equivalent to the following linear independence constraint qualification (LICQ):

$$
\nabla g_{i}\left(z^{*}\right), i \in I_{0} \cup I_{g}, \quad e^{i}, i \in I_{0}, \quad \text { are linearly independent. }
$$

Under this assumption, the following primal-dual necessary conditions hold:

$$
\begin{aligned}
\nabla f\left(z^{*}\right)= & \sum_{i \in I_{0} \cup I_{g}} \bar{\lambda}_{i} \nabla g_{i}\left(z^{*}\right)+\sum_{i \in I_{0}} \bar{\mu}_{i} e^{i}, \\
& \bar{\lambda}_{i} \geq 0, \quad \bar{\mu}_{i} \geq 0, \quad i \in I_{0} .
\end{aligned}
$$

To compare this result with ours, first note that LICQ (4.18) implies piecewise MFCQ, which is stronger than 2-regularity (recall Example 3.1). On the other hand, for our 
primal-dual conditions we need a tangent direction $\bar{h}$ which is critical (condition (4.3)). It is therefore clear that the two constraint qualifications are not directly comparable. In other words, one can be satisfied when the other is not, and vice versa. Secondly, note that primal-dual conditions (4.17) and (4.19) are also essentially different. If (3.14) holds (which is certainly implied by LICQ), as discussed above, we have that $\bar{\mu}^{2}=0$. Observe now that, since $I_{0}^{0} \cup I_{0}^{1}=I_{0}$, the right-hand side of (4.17) involves twice fewer gradients of constraints from the index set $I_{0}$ than (4.19). That is, in (4.17) the multipliers corresponding to half of the constraints in $I_{0}$ are claimed to be zero. In this respect, (4.17) is sharper than (4.19). On the other hand, (4.19) contains additional conditions on the signs of the multipliers in $I_{0}$. Hence, the two optimality conditions are essentially different, neither one of them being stronger than the other.

We next give some examples. Note that in the first example below, LICQ (4.18) holds, which is a much stronger condition than is needed to apply our results. Thus this example is certainly not the most suitable for showing the utility of our approach. We include it mainly because it is extensively used in the literature, e.g., [18]. Examples 4.2 and 4.3 better illustrate the differences and our contribution.

Example 4.1. Let $n=m=1$ and

$$
f(x, y)=\alpha x+\beta y, \quad g(x, y)=x,
$$

where $\alpha, \beta \in \Re$ are parameters. In the terminology of [18], the point $z^{*}=\left(x^{*}, y^{*}\right)=0$ is a $B$-stationary point (equivalent here to strongly stationary) if and only if $\alpha \geq 0$, $\beta \geq 0$. This is a primal stationarity condition. Concerning primal-dual stationarity, $z^{*}$ is a weak stationary point for any $\alpha$ and $\beta$; it is a $C$-stationary point if and only if either $\alpha \geq 0, \beta \geq 0$ or $\alpha \leq 0, \beta \leq 0$; and it is an $M$-stationary point if and only if $\alpha \geq 0, \beta \geq 0$ or $\alpha \beta=0$.

As is easy to see, $I_{0}=\{1\}, F^{\prime}\left(z^{*}\right)=0, H=\{(u, v) \mid u \geq 0, v \geq 0, u v=0\}$, and $F$ is 2-regular at $z^{*}$ with respect to all $(u, v) \in H \backslash\{0\}$ because $F^{\prime}\left(z^{*}\right)+P F^{\prime \prime}\left(z^{*}\right)[(u, v)]=$ $(v, u)$.

We start by illustrating our primal optimality conditions. If $\alpha>0, \beta>0$, then (4.2) holds, and the second assertion of Theorem 4.1 guarantees that $z^{*}$ is an isolated local minimizer. The first assertion of Theorem 4.1 shows that $z^{*}$ cannot be a minimizer unless $\alpha \geq 0, \beta \geq 0$. The latter is consistent with $B$-stationarity because in this example (3.14) holds, and $T_{D}\left(z^{*}\right)=\mathrm{cl} K=H=L$ (recall the comment after Theorem 4.1). Example 4.2 below shows that Theorem 4.1 is in fact useful beyond $B$-stationarity in the sense of [18].

Consider now primal-dual conditions.

Let $\alpha=0$ and $\beta>0$, in which case $z^{*}$ is a minimizer. Note that $\bar{h}=(\bar{u}, \bar{v})=(1,0)$ satisfies (4.3). We have that $I_{0}^{0}=\{1\}, I_{0}^{1}=\emptyset, \tilde{\lambda}_{1}=\bar{\lambda}_{1}\left\langle\nabla g\left(z^{*}\right), \bar{h}\right\rangle=\bar{\lambda}_{1}$, and (4.17) holds with $\tilde{\lambda}_{1}=-\beta$. All the stationarity conditions from [18] are also satisfied here.

Let $\alpha=1$ and $\beta=-1$, in which case $z^{*}$ is not a minimizer. In (4.3) we can take $\bar{h}=(\bar{u}, \bar{v})=(1,1)$, so that $I_{0}^{1}=\{1\}, I_{0}^{0}=\emptyset$, and $\tilde{\lambda}_{1}=\bar{\lambda}_{1} \bar{v}_{1}=\bar{\lambda}_{1}$. There exists no $\tilde{\lambda}_{1}$ such that $(1,-1)+\tilde{\lambda}_{1}(1,0)=0$, and so $(4.17)$ does not hold. Therefore this nonoptimal $z^{*}$ is not stationary in our sense. Note that it is weakly stationary but not $C$ - or $M$-stationary.

In the case $\alpha=0$ and $\beta<0$, the point $z^{*}$ is not a minimizer. But all the stationarity concepts of [18] hold, and it can be seen that Theorem 4.2 also applies. In this case, none of those stationarity concepts is able to detect nonoptimality of $z^{*}$.

The following examples are intended to show that the presented optimality conditions can be useful in situations where other approaches (e.g., $[15,18,6])$ do not apply. 
Example 4.2. Let $g, x^{*}$, and $y^{*}$ be as defined in Example 3.1 and the objective function be given by $f(x, y)=\alpha x+\beta_{1} y_{1}+\beta_{2} y_{2}+o(\|(x, y)\|)$, where $\alpha, \beta_{1}, \beta_{2}$ are parameters. Recall that not only (4.18) but even the much weaker condition (3.14) does not hold in this example. In particular, the constraint qualifications used in $[15,18]$ do not hold, and the corresponding results do not apply.

According to Example 3.1, the cone $H$ consists of four rays spanned by $(1,0,1)$, $(1,0,-1),(-1,1,1)$, and $(-1,1,-1)$, and the mapping $F$ is 2 -regular at $z^{*}$ with respect to every element in $H \backslash\{0\}$. From the primal necessary optimality conditions stated in Theorem 4.1, it can be derived that $z^{*}$ cannot be a local minimizer unless

$$
0 \leq \alpha \leq \beta_{1}, \quad\left|\beta_{2}\right| \leq \min \left\{a,\left|\beta_{1}-a\right|\right\} .
$$

Sufficient condition (4.2) is satisfied if and only if both of these inequalities hold strictly.

Next, for $\bar{h}=(1,0, \pm 1)$, equality in (4.3) takes place if and only if $\alpha \pm \beta_{2}=0$, and (4.4), (4.5) hold with $\bar{\lambda}=-\beta_{1}, \bar{\mu}^{1}=0, \bar{\mu}^{2}= \pm \beta_{2} / 2$. For $\bar{h}=(-1,1, \pm 1)$, equality in (4.3) takes place if and only if $-\alpha+\beta_{1} \pm \beta_{2}=0$, and (4.4), (4.5) hold with the same multipliers.

"Second-order" condition (4.7) does not provide any additional information in this example, as for every $\bar{h} \in H \backslash\{0\}$, the subspace comprised by elements $h$ satisfying (4.6) coincides with $\operatorname{span}\{\bar{h}\}$, and hence $B_{g}[h]^{2}=0$ for such $h$.

We next demonstrate the utility of the "second-order" condition (4.7). Note that this example also highlights the differences of the present paper as compared to optimality conditions in [6], where a general theory of 2-regularity is developed for $C^{1,1}$ mappings, and optimality conditions for MPEC are obtained after reformulating the constraints as $C^{1,1}$ equations.

Example 4.3. Let $n=m=2$,

$$
f(x, y)=x_{1}+y_{1}-y_{2}, \quad g_{1}(x, y)=x_{1}+y_{1}, \quad g_{2}(x, y)=x_{1}^{2}+x_{2}^{2}-\left(y_{2}-a\right)^{2},
$$

where $a>0$ is a parameter. Consider the point $z^{*}=\left(x^{*}, y^{*}\right) \in D$ with $x^{*}=0$, $y_{1}^{*}=0, y_{2}^{*}=a$. It can be verified that this $z^{*}$ is not a minimizer, but the stationarity conditions given in $[6$, section 6$]$ hold. Results from $[15,18]$ are not applicable (because $\nabla g_{2}\left(z^{*}\right)=0$ and thus constraint qualifications do not hold). We next show that Theorem 4.2 correctly classifies $z^{*}$ as nonoptimal.

We have that $I_{0}=\{1\}, I_{g}=\{2\}$,

$$
H=\left\{\begin{array}{l|l}
(u, v) & \begin{array}{l}
v_{1} \geq 0, u_{1}+v_{1} \geq 0, \\
v_{1}\left(u_{1}+v_{1}\right)=0 \\
u_{1}^{2}+u_{2}^{2}-v_{2}^{2}=0
\end{array}
\end{array}\right\} .
$$

It is easy to see that $F$ is 2-regular with respect to all elements $h=(u, v) \in H$ satisfying $u_{1} \neq 0$.

Take, e.g., $\bar{h}=(1,0,0,1) \in H$. Then equality in (4.3) is satisfied, and (4.4), (4.5) hold with $\bar{\lambda}=-1, \bar{\mu}^{1}=0, \bar{\mu}^{2}=-1$. Hence, $z^{*}$ is still a candidate for being optimal. A different analysis based on [6] leads to the same conclusion up to this point, and no further analysis is possible based on that reference.

However, using Theorem 4.2, we see that linear equalities (4.6) hold for elements $h=(u, v)$ such that $v_{1}=0, u_{1}-v_{2}=0$. For such $h$, it holds that

$$
\bar{\mu}^{2} B_{g}[h]^{2}=-u_{2}^{2}<0
$$


for any $u_{2} \neq 0$. Hence, condition (4.7) is violated here, and $z^{*}$ cannot be a local minimizer.

\section{REFERENCES}

[1] E.R. AvAKov, Extremum conditions for smooth problems with equality-type constraints, Comput. Math. Math. Phys., 25 (1985), pp. 24-32.

[2] E.R. Avakov, Necessary extremum conditions for smooth abnormal problems with equality and inequality constraints, Math. Notes, 45 (1989), pp. 431-437.

[3] J.F. Ben-Tal And J. Zowe, A unified theory of first- and second-order optimality conditions for extremum problems in topological vector spaces, Math. Programming Stud., 19 (1982), pp. 39-76.

[4] Y. Chen ANd M. Florian, The nonlinear bilevel programming problem: Formulations, regularity and optimality conditions, Optimization, 32 (1995), pp. 193-209.

[5] A.F. Izmailov, On certain generalizations of Morse's lemma, Proc. Steklov Inst. Math., 220 (1998), pp. 138-153.

[6] A.F. Izmailov and M.V. Solodov, The theory of 2-regularity for mappings with Lipschitzian derivatives and its applications to optimality conditions, Math. Oper. Res., (2002), to appear.

[7] A.F. Izmailov AND M.V. Solodov, Error bounds for 2-regular mappings with Lipschitzian derivatives and their applications, Math. Program., 89 (2001), pp. 413-435.

[8] A.F. Izmailov and M.V. Solodov, Optimality conditions for irregular inequality-constrained problems, SIAM J. Control Optim., 40 (2001), pp. 1280-1295.

[9] U. LEDZEWICZ AND H. SCHÄTTLER, High-order approximations and generalized necessary conditions for optimality, SIAM J. Control Optim., 37 (1998), pp. 33-53.

[10] Z.-Q. Luo, J.-S. PAng, And D. RAlPh, Mathematical Programs with Equilibrium Constraints, Cambridge University Press, Cambridge, UK, 1996.

[11] Z.-Q. LuO, J.-S. PANG, D. RALPH, AND S.-Q. Wu, Exact penalization and stationarity conditions of mathematical programs with equilibrium constraints, Math. Programming, 75 (1996), pp. 19-76.

[12] R.J. MaGnus, On the local structure of the zero-set of a Banach space valued mapping, J. Funct. Anal., 22 (1976), pp. 58-72.

[13] O.L. Mangasarian and S. Fromovitz, The Fritz John necessary optimality conditions in the presence of equality and inequality constraints, J. Math. Anal. Appl., 7 (1967), pp. 37-47.

[14] J.V. Outrata, Optimality conditions for a class of mathematical programs with equilibrium constraints, Math. Oper. Res., 24 (1999), pp. 627-644.

[15] J.-S. PANG AND M. FukUShima, Complementarity constraint qualifications and simplified Bstationarity conditions for mathematical programs with equilibrium constraints, Comput. Optim. Appl., 13 (1999), pp. 111-136.

[16] J.-P. PEnot, Second-order conditions for optimization problems with constraints, SIAM J. Control Optim., 37 (1999), pp. 303-318.

[17] S.M. Robinson, Strongly regular generalized equations, Math. Oper. Res., 5 (1980), pp. 43-62.

[18] H. Scheel AND S. Scholtes, Mathematical programs with complementarity constraints: Stationarity, optimality and sensitivity, Math. Oper. Res., 25 (2000), pp. 1-22.

[19] A.A. TRETYAKov, Necessary and sufficient conditions for optimality of pth order, Comput. Math. Math. Phys., 24 (1984), pp. 123-127.

[20] J.J. YE, Optimality conditions for optimization problems with complementarity constraints, SIAM J. Optim., 9 (1999), pp. 374-387. 